\title{
Fluconazole and acetazolamide in the treatment of ectopic Cushing's syndrome with severe metabolic alkalosis
}

\author{
Verena Schwetz, Felix Aberer, Claudia Stiegler, Thomas R Pieber, \\ Barbara Obermayer-Pietsch and Stefan Pilz
}

Clinical Division of Endocrinology and Metabolism, Department of Internal Medicine, Medical University of Graz, Graz, Austria
Correspondence

should be addressed

to V Schwetz

Email

verena.schwetz@medunigraz.at

\section{Summary}

Cushing's syndrome (CS) due to ectopic ACTH production accounts for about $10 \%$ of all types of CS and is frequently associated with metabolic alkalosis. Treatment of CS involves surgical resection and/or medical therapy to control hypercortisolism. We present the case of an 80-year-old woman affected by CS due to an unknown cause. The patient had severe metabolic alkalosis with refractory hypokalemia. To treat the underlying CS, fluconazole was initiated due to unavailability of ketoconazole. In spite of markedly decreasing cortisol levels, metabolic alkalosis persisted. Treatment of metabolic alkalosis with acetazolamide was thus initiated and pH levels successfully lowered. This case report shows that hypercortisolism can be effectively treated with fluconazole in cases where ketoconazole is unavailable or not tolerated and that persistent severe metabolic alkalosis caused by glucocorticoid excess can be safely and successfully treated with acetazolamide.

\section{Learning points:}

- Hypercortisolism can be effectively treated with fluconazole where ketoconazole is unavailable or not tolerated.

- Glucocorticoid excess can cause severe metabolic alkalosis.

- Persistent severe metabolic alkalosis can be safely and successfully treated with acetazolamide.

\section{Background}

Ectopic adrenocorticotropic hormone (ACTH) syndrome accounts for about $10 \%$ of all types of Cushing's syndrome (CS). Bronchial carcinoid tumors are the most common causes of ectopic ACTH secretion, followed by neuroendocrine tumors, whereas in $12.5 \%$ the source of ACTH production is never found (1). The clinical features of hypercortisolemia in ectopic CS usually are of rapid onset and progression. Biochemical characteristics include anemia, reduced white blood count, hypokalemia and elevated levels of bicarbonate (1) causing metabolic alkalosis through the mineralocorticoid effect of cortisol.
Treatment options include surgical excision of the tumor, if possible, and/or medical therapy. Medical options include adrenal-directed agents such as ketoconazole, metyrapone, mitotane, pituitary-directed agents such as dopamine agonists and somatostatin analogues and the glucocorticoid-receptor-directed agent mifepristone. Case reports in critically ill patients receiving high-dose fluconazole have shown that fluconazole might suppress adrenal steroidogenesis as well (defined as peak cortisol level after ACTH stimulation). (2) Reports documenting the use of fluconazole in CS are scarce; one case report, 
however, shows long-term control of hypercortisolism in a patient with pulmonary metastases due to an adrenal glucocorticoid-producing tumor. (3) In 154 critically ill surgical patients without overt CS, Magill et al. (4), did not detect a significant difference in mean plasma cortisol levels in patients randomized to fluconazole when compared to placebo. Nonetheless, fluconazole might be considered a treatment option in selected CS cases where ketoconazole is not tolerated or unavailable. Fluconazole might even be less toxic than ketoconazole, even though improved safety with respect to liver injury is not clearly established (5).

In patients with ectopic CS and metabolic alkalosis, the treatment of the underlying cause, i.e. controlling of hypercortisolism, is the treatment of choice because metabolic alkalosis can emerge as a consequence of mineralocorticoid receptor activation in states of cortisol excess. In this context, it is important to note that metabolic alkalosis occurring from other causes such as loop diuretic therapy can be controlled by administering acetazolamide, a carbonic anhydrase inhibitor with diuretic effects (6). Its use in treating metabolic alkalosis in CS has, to the best of our knowledge, not been described in the literature so far.

We therefore present the case of an 80-year-old woman affected by CS with severe metabolic alkalosis and refractory hypokalemia. To treat the underlying CS and cause of metabolic alkalosis, while failing to locate the site of ACTH production, therapy with fluconazole was initiated due to unavailability of ketoconazole. In spite of markedly decreasing cortisol levels, metabolic alkalosis persisted. Treatment of metabolic alkalosis with acetazolamide was thus initiated.

\section{Case presentation}

An 80-year-old woman was admitted to hospital for evaluation of vertigo and extreme fatigue and muscle weakness lasting for several weeks. Clinical evaluation revealed candida albicans stomatitis and extreme fatigue. Medical history revealed chronic lymphocytic leukemia, hypertension, osteoporosis and stroke. Her initial medication included clopidogrel, antihypertensive agents, a proton pump inhibitor, a bisphosphonate and thyroid hormone. Easy bruising, facial plethora, proximal muscle weakness or striae were not noted; neither were hirsutism nor centripetal obesity. Upon admission, new onset hyperglycemia, hypokalemia and metabolic alkalosis were detected. Hypokalemia was refractory to potassium substitution and even spironolactone. Endocrinological work-up revealed elevated levels of cortisol and ACTH, the other hormone levels were normal, apart from a deficiency in growth hormone and insulin-like growth factor 1 .

\section{Investigation}

The presence of CS was confirmed on the basis of a lack of suppression of serum cortisol after a low-dose (1 mg) dexamethasone suppression test (cortisol $1049.2 \mathrm{ng} / \mathrm{ml}$, ACTH $29.5 \mathrm{pg} / \mathrm{ml}$ ), a high-dose (8 mg) dexamethasone suppression test, as well as elevated 24-h-urinary cortisol (112.0 $\mu \mathrm{g} / 24 \mathrm{~h}$, reference range 3.0-43.0).

The suspicion of an ectopic source of ACTH was supported by the short onset of clinical symptoms, by the presence of high values of ACTH and the lack of serum cortisol suppression after a high-dose dexamethasone suppression test. Corticotropin-releasing hormone (CRH) stimulation, however, showed increases of serum cortisol and ACTH, indicative of Cushing's disease (cortisol: $601.7 \mathrm{ng} / \mathrm{ml}$ (baseline) to $871.2 \mathrm{ng} / \mathrm{ml}$ (15 $\mathrm{min}$ ) to $458.5 \mathrm{ng} / \mathrm{ml}$ (30 min); ACTH from $378 \mathrm{pg} / \mathrm{ml}$ (baseline) to $491 \mathrm{pg} / \mathrm{ml}(15 \mathrm{~min})$ to $149 \mathrm{pg} / \mathrm{ml}$ (30 $\mathrm{min}))$. Yet magnetic resonance imaging of the sella did not reveal adenoma, which does not, however, entirely exclude the possibility of a small ACTH-producing lesion in the pituitary gland. Computed tomography scan (CT) of the abdomen showed hypertrophy of the adrenal glands on both sides.

To locate the source of ACTH production, CT of the thorax was performed which revealed a known $8 \mathrm{~mm}$ lesion in the right upper lobe of the lung. As the lesion had been stable over years, it was not considered a likely source of ACTH production, especially since octreoscan, F-18 DOPA PET-CT as well as FDG-PET were inconspicuous. The source of ectopic ACTH production was not found. Petrosal venous sinus sampling could not be performed due to the patient's poor general state of health.

\section{Treatment}

While performing the diagnostic work-up, medical therapy to treat hypercortisolism was initiated. As ketoconazole and metyrapone were unavailable, fluconazole was administered, causing a substantial decrease in cortisol levels (Fig. 1A). Bilateral adrenalectomy was not an option due to the patient's poor general health condition. Fluconazole was started with $200 \mathrm{mg}$ daily, after 2 days was increased to $400 \mathrm{mg}$, then gradually up to $1200 \mathrm{mg}$. After 1 week, fluconazole doses were tapered again to 

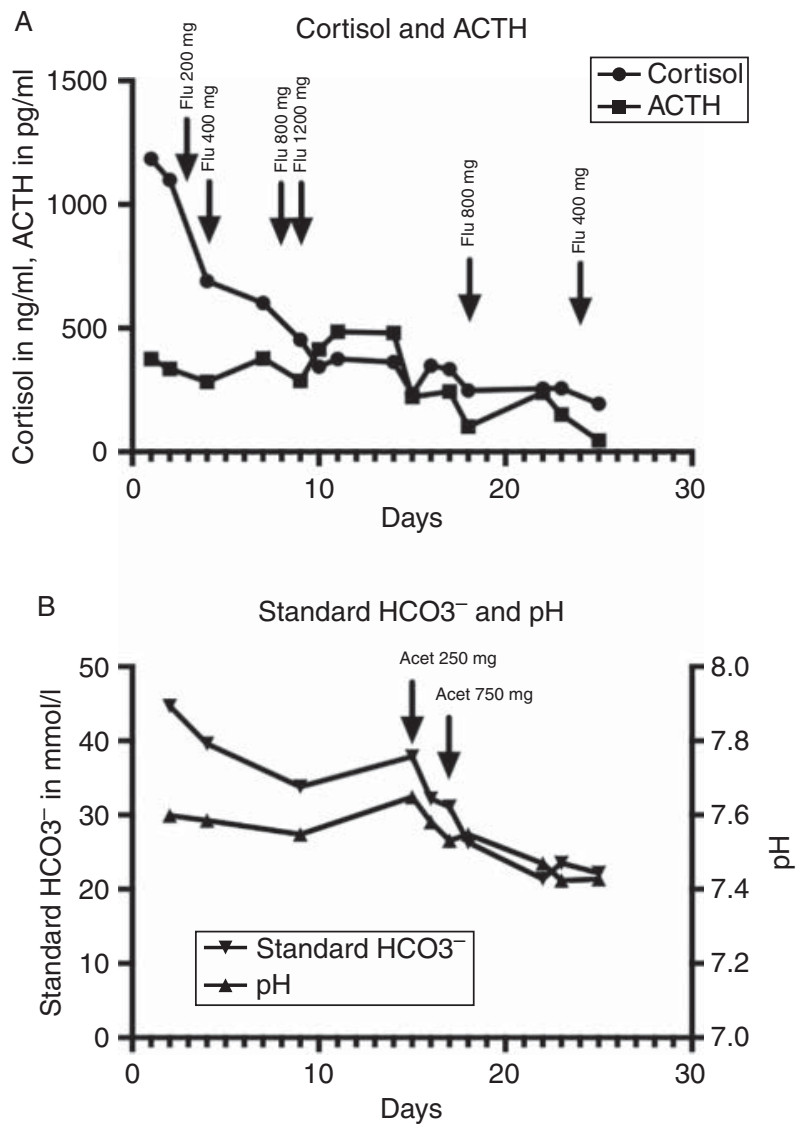

Figure 1

(A) Changes of cortisol and ACTH in response to treatment with fluconazole (Flu). Reference ranges: cortisol $43.0-220.0 \mathrm{ng} / \mathrm{ml}$, ACTH $10.0-51.0 \mathrm{pg} / \mathrm{ml}$. Treatment changes: Day 3 Fluconazole $200 \mathrm{mg}$, day 4 fluconazole $400 \mathrm{mg}$, day 8 fluconazole $800 \mathrm{mg}$, day 9 fluconazole $1200 \mathrm{mg}$, day 10 fluconazole $1200 \mathrm{mg}$, day 18 fluconazole $800 \mathrm{mg}$, day 24 fluconazole $400 \mathrm{mg}$. (B) Changes in $\mathrm{pH}$ and standard bicarbonate in response to treatment with acetazolamide (Acet). Reference ranges: pH 7.35-7.45, standard bicarbonate $22-26 \mathrm{mmol} / \mathrm{l}$. Treatment changes: Day 15 acetazolamide $250 \mathrm{mg}$, day 17 acetazolamide $750 \mathrm{mg}$.

$400 \mathrm{mg}$. Cortisol levels remained in the upper limit of normal despite dose reduction.

Even though the patient's vigilance improved markedly and other causes of metabolic alkalosis had been ruled out, $\mathrm{pH}$ levels, i.e. severe metabolic alkalosis, remained unchanged after cortisol decrease. To treat metabolic alkalosis, therapy with acetazolamide was started at an initial dose of $250 \mathrm{mg}$ and was gradually increased to $750 \mathrm{mg} /$ day (Fig. 1B). pH levels normalized under this treatment. Upon the gradual decrease of cortisol levels, but more upon normalization of metabolic alkalosis, potassium levels returned to normal levels and less substitution and treatment with spironolactone were necessary.
After 12 days of fluconazole treatment, liver enzymes started rising (in total, alkaline phosphatase rose from 79 to $1293 \mathrm{U} / 1$ (35-105), gamma-glutamyl transferase from 106 to $891 \mathrm{U} / 1$ (-38), aspartate transaminase from 27 to $57 \mathrm{U} / \mathrm{l}(-30)$, alanine transaminase stayed in the normal range and cholinesterase decreased from 2499 to $978 \mathrm{U} / \mathrm{l}$ (3900-11000)). Bilirubine, albumin and prothrombin time remained in the normal range, liver failure did not develop. Other causes of cholestasis were ruled out.

\section{Outcome and follow-up}

During the patient's stay in the hospital, she developed pancytopenia. Bone marrow biopsy was carried out, revealing myelodysplasia. Staining the biopsy for ACTH or CRH did not show any positivity. Clinical deterioration ensued, culminating in the patient's death approximately one month after diagnosis.

\section{Discussion}

This case report shows that hypercortisolism can be effectively treated with fluconazole in cases where ketoconazole is unavailable or not tolerated and that persistent severe metabolic alkalosis caused by glucocorticoid excess can be safely and successfully treated with acetazolamide.

This case report of a woman with ectopic ACTH production, the source of which could not be found, shows that fluconazole effectively normalizes cortisol levels and could thus be an alternative when either ketoconazole is unavailable or not tolerated by the patient. The only published paper on fluconazole treatment in CS reports of a patient with a history of a tumor of the right adrenal gland that was initially successfully operated. Approximately 3.5 years later, this patient had developed lung metastases and was subsequently successfully treated with fluconazole at a dose of $400 \mathrm{mg}$ daily for 18 months. In the same publication, the authors describe another unpublished case of a patient with ACTHdependent CS who initially responded well to fluconazole (with a 66\% reduction) but showed an increase thereafter. In our patient, cortisol levels dropped by about $82 \%$ and remained low in spite of dose reduction of fluconazole to $400 \mathrm{mg}$ daily. The question of long-term cortisol normalization, however, remains unanswered.

Reviews on antifungal agents claim that fluconazole, in contrast to ketokonazole, does not interfere with adrenocortical function (7) (8), while other authors report on adrenal dysfunction after fluconazole ranging from 
mild impairment to severe insufficiency (2) (9). Interestingly, and in contrast to our understanding of fluconazole's mechanism of action and to what is described in the case report by Albert (2), ACTH levels in the patient we describe did not significantly increase.

A communication of the United States Food and Drug Administration states that the overall risk for ketoconazole-induced serious hepatic injury appears to be higher than that associated with other azole agents (5). A recent review comes to the conclusion, however, that the current literature does not allow a clear judgment of whether fluconazole carries a lower risk for hepatic injury than ketoconazole (5). In our patient, fluconazole was well tolerated even at doses up to $1200 \mathrm{mg}$ for a short period of time, even though liver enzymes did increase. The period of observation was too short to determine whether the dose reduction would have caused a decrease of the elevated liver enzymes.

As with long-term efficacy, questions of long-term safety remain currently unanswered. Fluconazole might be an option to treat CS due to adrenal cortisol production but also ACTH-dependent CS. For the short period of observation of 3 weeks, efficacy and tolerability were good, implying fluconazole as a medical treatment option in cases where ketoconazole is unavailable or not tolerated, even in cases of ACTH-dependent CS. This is supported by in vitro data showing inhibition of human adrenocortical steroidogenesis by fluconazole (10).

The effect of fluconazole was monitored with morning serum cortisol levels $(800 \mathrm{~h})$. These values do not ideally reflect the activity of the tumor, but could easily and quickly be measured. Importantly, morning serum cortisol levels gradually decreased as doses of fluconazole were increased. This decrease in cortisol levels was paralleled by declining glucose levels along with reduced requirement of insulin doses and, most importantly, improved patient's general condition.

In spite of decreasing cortisol and glucose levels, metabolic alkalosis did not improve. However, no other cause for metabolic alkalosis could be detected. Metabolic alkalosis in CS is caused by mineralocorticoid action of excess glucocorticoids and is associated with increased mortality. In a study of 409 medical and surgical patients with metabolic alkalosis, the overall mortality was $27.9 \%$ (11). Mortality increased as $\mathrm{pH}$ values rose, reaching $48.5 \%$ when the $\mathrm{pH}$ was greater than 7.60 . Patients with bicarbonate levels up to $40 \mathrm{mmol} / \mathrm{l}$ are usually asymptomatic (12). When symptoms do occur, they are mostly related to the accompanying hypokalemia (12). As bicarbonate levels increase to above $50 \mathrm{mmol} / \mathrm{l}$, however, patients can develop seizures, altered mental status or coma (12).

As $\mathrm{pH}$ levels were persistently high in our patient in spite of decreasing cortisol levels and the patient's clinical condition was still poor in terms of vigilance, treatment with the carbonic anhydrase inhibitor acetazolamide was initiated. Acetazolamide is a carbonic anhydrase inhibitor causing alkaline diuresis (6). The dose was started with $250 \mathrm{mg}$ and augmented to $750 \mathrm{mg}$. pH levels successfully returned to normal under this treatment. To the best of our knowledge, the use of acetazolamide in the treatment of metabolic alkalosis in CS has not been described in the literature so far. In the case we describe, the use of acetazolamide was safe, $\mathrm{pH}$ decreased and clinical condition improved.

Indeed, as the site of ACTH production could not be localized, treatment with fluconazole and acetazolamide were of symptomatic nature. They were, however, successful in normalizing cortisol and $\mathrm{pH}$ levels, in improving hyperglycemia and in ameliorating the patient's clinical condition at least temporarily.

Declaration of interest

The authors declare that there is no conflict of interest that could be perceived as prejudicing the impartiality of the research reported.

\section{Funding}

This research did not receive any specific grant from any funding agency in the public, commercial or not-for-profit sector.

\section{Patient consent}

The patient is dead. We managed to contact the patient's 85-year-old husband and obtained an oral consent to publish this case report.

\section{Author contribution statement}

V Schwetz and S Pilz were involved in patient care and preparation of the manuscript. F Aberer, C Stiegler and T R Pieber were involved in patient care. B Obermayer-Pietsch carried out analysis of laboratory parameters.

\section{References}

1 Isidori AM, Kaltsas GA, Pozza C, Frajese V, Newell-Price J, Reznek RH, Jenkins PJ, Monson JP, Grossman AB \& Besser GM 2006 The ectopic adrenocorticotropin syndrome: clinical features, diagnosis, management, and long-term follow-up. Journal of Clinical Endocrinology and Metabolism 91 371-377. (doi:10.1210/jc.2005-1542)

2 Albert SG, DeLeon MJ \& Silverberg AB 2001 Possible association between high-dose fluconazole and adrenal insufficiency in critically ill patients. Critical Care Medicine 29 668-670. (doi:10.1097/00003246200103000-00039) 
3 Riedl M, Maier C, Zettinig G, Nowotny P, Schima W \& Luger A 2006 Long term control of hypercortisolism with fluconazole: case report and in vitro studies. European Journal of Endocrinology 154 519-524. (doi:10.1530/eje.1.02120)

4 Magill SS, Puthanakit T, Swoboda SM, Carson KA, Salvatori R, Lipsett PA \& Hendrix CW 2004 Impact of fluconazole prophylaxis on cortisol levels in critically ill surgical patients. Antimicrobial Agents and Chemotherapy 48 2471-2476. (doi:10.1128/AAC.48.7.2471-2476.2004)

5 Greenblatt HK \& Greenblatt DJ 2014 Liver injury associated with ketoconazole: review of the published evidence. Journal of Clinical Pharmacology 54 1321-1329. (doi:10.1002/jcph.400)

6 Kassamali R \& Sica DA 2011 Acetazolamide: a forgotten diuretic agent. Cardiology in Review 19 276-278. (doi:10.1097/CRD.0b013e31822b4939)

7 Como JA \& Dismukes WE 1994 Oral azole drugs as systemic antifungal therapy. New England Journal of Medicine 330 263-272. (doi:10.1056/ NEJM199401273300407)
8 Terrell CL 1999 Antifungal agents, Part II. the azoles. Mayo Clinic Proceedings 74 78-100. (doi:10.4065/74.1.78)

9 Shibata S, Kami M, Kanda Y, Machida U, Iwata H, Kishi Y, Takeshita A, Miyakoshi S, Ueyama J, Morinaga S et al. 2001 Acute adrenal failure associated with fluconazole after administration of high-dose cyclophosphamide. American Journal of Hematology 66 303-305. (doi:10.1002/ajh.1063)

10 van der Pas R, Hofland LJ, Hofland J, Taylor AE, Arlt W, Steenbergen J, van Koetsveld PM, de Herder WW, de Jong FH \& Feelders RA 2012 Fluconazole inhibits human adrenocortical steroidogenesis in vitro. Journal of Endocrinology 215 403-412. (doi:10.1530/JOE-12-0310)

11 Galla JH 2000 Metabolic alkalosis. Journal of the American Society of Nephrology 11 369-375.

12 Soifer JT \& Kim HT 2014 Approach to metabolic alkalosis. Emergency Medicine Clinics of North America 32 453-463. (doi:10.1016/j.emc.2014. $01.005)$

Received in final form 15 May 2015

Accepted 21 May 2015 